\title{
The Impacts of an Educational Program by Using Group Discussion on Perceived Stigma Among the Family Caregivers of People with
} Alzheimer's Disease

\author{
Mahboobeh Alipour Chermahini ${ }^{1}$, Soroor Parvizy (iiD) ${ }^{2}$, Fatemeh Hajibabaee ${ }^{1}$, Elham Navab (iD ${ }^{1,{ }^{*}}$ and \\ Hamid Haghani (ib $^{3}$ \\ ${ }^{1}$ School of Nursing \& Midwifery School, Tehran University of Medical Sciences. Tehran, Iran \\ ${ }^{2}$ School of Nursing \& Midwifery, Iran University of Medical Sciences, Tehran, Iran \\ ${ }^{3}$ Iran University of Medical Sciences, Tehran, Iran \\ "Corresponding author: School of Nursing \& Midwifery, Tehran University of Medical Sciences, Tehran, Iran. Email: e_navab100@hotmail.com
}

Received 2021 March 06; Revised 2021 April 24; Accepted 2021 May 09.

\begin{abstract}
Background: The objective of the present study was to determine the impacts of an educational program by using group discussion on perceived stigma among family caregivers of people with Alzheimer's disease (AD).

Methods: This nonequivalent non-randomized controlled trial was performed in 2015. A sample of 66 family caregivers of people with $\mathrm{AD}$ was recruited conveniently. The caregivers were non-randomly allocated to a control and an experimental group. Initially, the perceived stigma of all participants was measured by using the Stigma Impact Scale. Then a five-session educational program by using group discussion was held over five weeks for the caregivers in the experimental group. The caregivers in the control group received no intervention. The level of caregivers' perceived stress was re-assessed both immediately and one month after the intervention.

Results: The difference between the groups in terms of pretest perceived stigma was not statistically significant $(\mathrm{P}=0.146)$. However, immediately and one month after the intervention, the mean score of perceived stigma in the experimental group was significantly lower than in the control group $(\mathrm{P}<0.05)$. The results of repeated measures ANOVA for the between-subject factor of the group also showed that the intervention had significant effects on the caregivers' perceived stigma $(\mathrm{P}=0.004)$.

Conclusions: The findings of the present study showed the noticeable effects of the educational program using group discussion on the perceived stigma of family caregivers of people with AD. This finding can denote the importance of experience sharing in the psychological well-being of family caregivers. Through group discussion and experience sharing, caregivers learn the necessary skills for coping with their own problems. Given their effectiveness, educational interventions by using group discussion are recommended to reduce perceived stigma among family caregivers.
\end{abstract}

Keywords: Alzheimer's Disease, Stigma, Family Caregivers, Educational Program, Group Discussion

\section{Background}

Alzheimer's disease (AD) is a chronic progressive and debilitating brain disorder which has profound effects on memory, intelligence, cognition, speech, physical functioning, activities of daily living, and orientation to the place and person, as well as self-care, planning, innovation, organization, and abstract reasoning abilities. Epidemiological studies on the elderly confirm a pandemic of $\mathrm{AD}$ (1). According to the statistics provided by the World Health Organization (WHO), the number of people with dementia in the world was 35.6 million in 2012, which is estimated to reach 65.7 and 115.4 million by 2030 and 2050, respectively
(2).

Due to cognitive and behavioral alterations, people with $\mathrm{AD}$ are unable to fulfill their own needs, and hence, they are greatly dependent on the help of others for fulfilling their needs (3), denoting the fact that the most important source of caregiving to people with $\mathrm{AD}$ is the family (4). Given the need of people with AD for long-term care services, their family caregivers are indisputably at risk for relatively high levels of stress, strain, and psychological problems compared with the family members of elders with physical health problems $(5,6)$.

Goffman (7) considered stigma a spoiled identity at which a characteristic is attributed to a person or a group, 
so the person or the members of that group are devalued and differentiated and are no longer considered healthy and competent individuals. Stigma can cause patients/caregivers to hide the illness (8). Therefore, AD family caregivers usually suffer from lack of social support and relationships and experience a sense of social isolation. They gradually move toward sacrificing their own leisure times, limiting their relationships with friends and relatives, and leaving their employment (9). For instance, in one of a few quantitative studies dealing with AD family caregivers' stigma, it was noted that adult children (50\%), spouses (40\%), and other family caregivers (10\%) experienced stigma (10).

Family stigma is caused by a degree of unusualness in the family, which can differ by society and culture (11). In the collectivist culture of Iran, family members are less concerned about the negative effects of mental disorders on themselves than about their reputation and how others view them. Thus, given the negative implications of stigma for the mental health of the family and the importance of the family in giving continuous care and treatment to AD patients, the phenomenon is considered a major issue and requires further investigations.

One of the strategies to improve family caregivers' ability to provide care to their ill members is education. Family caregivers need to receive education about the underlying conditions of their ill members and how to give care to them (12). Patient and family education is one of the most basic responsibilities of healthcare providers, particularly nurses, the fastest and the most effective strategy to attain the goal of social justice, and a key strategy for supporting family caregivers and improving their condition (13).

In one study on AD family caregivers, a combination method was used by Liu, indicating that the more severe the perceived stigma, the stronger the feeling of depression (14). Despite the large number of AD patients in Iran, there is just one service provider organization [i.e., Iran Alzheimer Association (IAA)] because there are obstacles to funding and organizing such foundations. Likewise, there are limited support services for Iranian families caring for a person with $\mathrm{AD}$.

\section{Objectives}

The present study aimed to determine the impacts of an educational program based on group discussions on perceived stigma among AD family caregivers.

\section{Methods}

\subsection{Study Design, Setting, and Subjects}

This was a nonequivalent non-randomized controlled trial performed in summer 2015 (Figure 1).

Study setting was the Iranian Alzheimer's Association located in Ekbatan, Tehran, Iran, and the study population were the family caregivers of people with AD. Based on the results of a previous study and considering a standard deviation of 11.00 (14), the sample size for comparing two means was designated as 66 using a formula. Consequently, using a convenient sampling method, 66 caregivers were recruited.

Inclusion criteria were being the main caregiver of the person, giving care for at least six months, having no hearing problems, being able to read and perceive educational materials and answer study items, agreeing to participate in the program, actively participating in group discussions, and speaking Persian. Exclusion criteria were the death of the afflicted person, the change of the main family caregiver, and failure to participate in more than one educational session. The participants were non-randomly allocated to the experimental and control groups. In order to prevent the leak of information from one group to another, we initially recruited all the caregivers of the control group and then started to recruit caregivers for the experimental group.

\subsection{Instruments}

Data collection instruments were a demographic questionnaire and the Stigma Impact Scale (SIS). The items of the demographic questionnaire were gender, age, educational and marital status, AD stage, number of caregivers, the kinship of the main caregiver with the afflicted person, the place where the afflicted person lived, the time spent per day on caregiving, the total duration of caregiving, the type of insurance coverage, the caregiver's employment status, and the financial status of the family. This questionnaire was filled by all the participants during a general session held before initiating the study intervention.

The SIS measured perceived stigma in the caregivers of persons with AD. This scale was used and validated by Liu et. al. (14). The SIS comprises 24 items assessing AD family caregivers' perceptions of stigma in the four dimensions of social rejection (nine items), financial insecurity (three items), internalized shame (five items), and social isolation (seven items). The items are responded to and scored as follows: (1) no idea, 0; (2) completely disagree, 1; (3) disagree, 2; (4) agree, 3; and (5) completely agree, 4 . The total score could range from 0 to 96 ; the closer the score to 96 , the higher the perceived stigma (12). 


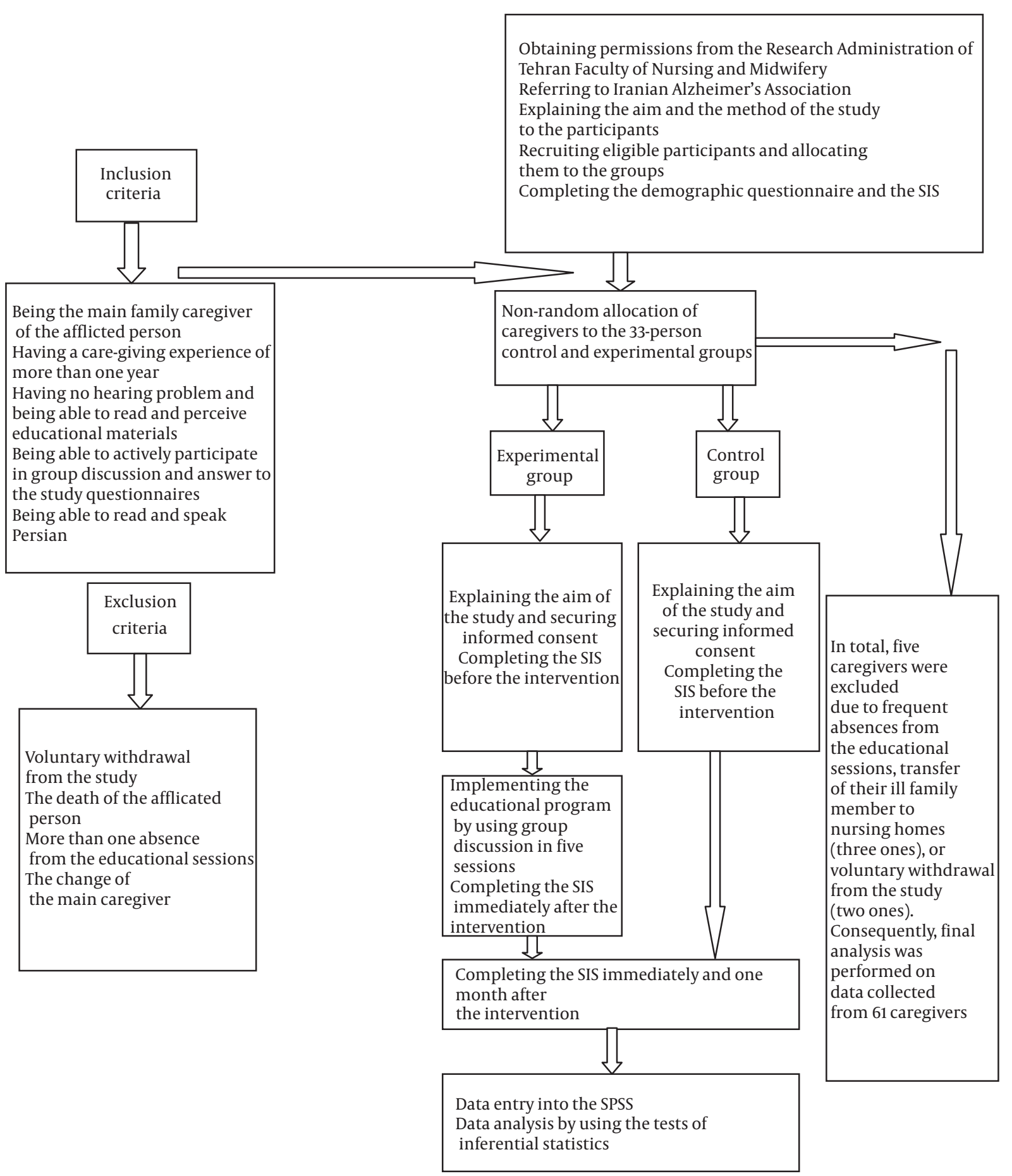

Figure 1. The flow diagram of the study 
In this study, to ensure the scientific validity of the SIS, the content validity technique was applied. Accordingly, for internal validity, the multiple-forward translation method (15) was utilized to translate the SIS from the source language (English) into the target language (Persian). Two researchers translated each section; the two translations were compared and also joined to obtain the most appropriately translated version, which was finally approved by the main researcher in terms of scientific and cultural validity. The content validity of the instrument was approved by 10 faculty members of Tehran University of Medical Sciences, Faculty of Nursing and Midwifery, and its reliability was assessed via the test-retest method. Accordingly, 10 caregivers filled out the scale twice with an interval of two weeks. The correlation coefficient between the test and retest scores was 0.7 , showing a satisfactory reliability.

\subsection{Intervention}

One week before holding educational sessions, we held an inaugural session to explain the aim and process of the study to the participants and also to ask them to complete the demographic questionnaire and the SIS (i.e., pretest). Then a five-session educational program was held for the caregivers in the experimental group that had been already divided into 8 - 10-member subgroups. The sessions were held weekly based on the group discussion technique. The research team attended all the sessions, and the group was guided by the researcher and professors. The meetings were held at the Alzheimer's Association center in a calm and convenient environment.

The length of each session was about 90 minutes. Group discussion is one of the active teaching-learning methods and an organized dialogue among all group members about a topic of interest. Group discussion provides group members with the opportunity to share their ideas, beliefs, and experiences (9). In the first session, the caregivers became familiar with each other and provided a brief history of their ill family members' condition. Then the aim and process of the study were briefly explained, and a timetable was given to the participants, containing the titles, dates, and times of the sessions. Moreover, the importance of family's role in giving care to people with $\mathrm{AD}$ was discussed. In the second session, a recreational tour was held for the participants to familiarize themselves with each other, strengthen their relationships and interactions, boost their trust in each other, and thereby facilitate their active participation in subsequent group discussions. In the third and fourth sessions, the researchers and participants discussed $\mathrm{AD}$, its prognosis, the behavioral problems of afflicted persons, stigma and its types, family caregivers' experiences of stigma, strategies to prevent or reduce stigmatization, coping skills, and strategies to manage tensions and feelings. In these two sessions, the participants were also asked to share their own ideas and experiences of caregiving. In the fifth session, conclusions were drawn, and the participants were asked to share their experiences of using coping skills and feelingmanagement strategies in the face of stigmatization. Finally, a booklet containing the educational materials was given to each participant. Immediately and one month after implementing the program, perceived stigma was reassessed using the SIS tool in both groups. So, perceived stigma was measured thrice, namely before the intervention (T1), immediately after it (T2), and one month later (T3).

\subsection{Ethical Considerations}

This study was approved by the Institutional Review Board of the Tehran University of Medical Sciences, Tehran, Iran (IRB approval number: 94S250802) and registered in the Iranian Registry of Clinical Trials (IRCT201502047212N6). We provided the participants with information about the aims and importance of the study and ensured them about the confidentiality of the study data and the fact that they were free to decide to participate or not. Then verbal and written informed consent was secured (Figure 1). Additionally, after the posttest, we provided the caregivers in the control group with educational materials.

\subsection{Data Analysis}

Data were analyzed using SPSS-20 software and descriptive statistics (mean, standard deviation, and frequency tables) to present the data. Inferential statistics [chi-Square, Fisher's exact test, and repeated measures variance test (RM-ANOVA)] were employed to compare stigma scores before, immediately after, and one month after the intervention, and Bonferroni's post hoc test was utilized for pairwise comparisons.

\section{Results}

The means of the caregivers' age in the experimental and control groups were $52 \pm 11.44$ and $49.8 \pm 12$ years $(\mathrm{P}=$ $0.464)$, respectively. Most participants in both groups were female, and housewives held diploma degrees and gave care to people with third-stage AD. Moreover, they were mostly the daughters of people with AD (Table 1 ). The results of the Fisher's exact test and the independent-sample $t$-test illustrated no significant between-group differences with regard to the participants' gender, age, education, 
marital status, AD stage, the number of family caregivers, main caregiver, caregiver's kinship with the afflicted person, the afflicted person's place of living, the time spent per day on caregiving, the total duration of caregiving, the type of insurance coverage, and the financial status of the family.

The independent-sample $t$-test indicated that the difference between the groups in terms of perceived stigma was not statistically significant at the pretest phase $(\mathrm{P}=$ 0.146). However, immediately and one month after the intervention, there were significant differences between the groups in terms of perceived stigma score $(\mathrm{P}<0.051)$. The results of RM-ANOVA for the between-subject factor of the group also showed that the intervention had significant effects on caregivers' perceived stigma $(P=0.004)$. Bonferroni's post hoc test revealed a significant difference between the T1 and T2, as well as between T1 and T3 phases in both groups with regard to the mean scores of perceived stigma $(\mathrm{P}<0.001)$. The trends of variations were downward in both groups (Figure 2). Although perceived stigma decreased after the study period in both groups, the rate of decrease in the experimental group was greater. Nonetheless, the groups did not significantly differ from each other in terms of the pretest-posttest mean difference of perceived stigma score $(P=0.412$, Table 2$)$.

\section{Discussion}

Stigma is an important issue for patients with chronic mental disorders such as AD. The objective of this research was to determine the impacts of a group discussion-based educational program on perceived stigma among AD family caregivers. Our findings revealed a significant betweengroup difference comparing the mean scores of perceived stigma both immediately and one month after the intervention, implying the effectiveness of the educational program in mitigating AD caregivers' perceived stigma.

Uchino et al. (2012) and Vaghei et al. (2015) showed that psych education was effective in reducing the stigma perceived by patients with chronic mental disorders and their family caregivers $(16,17)$. The results of a study conducted by Griffiths et al. (2014) suggested that interventions had negligible effects on personal stigma and social distancing while they significantly reduced stigma in relation to chronic mental disorders such as Alzheimer's. It was also found that educational interventions were as effective as face-to-face communication (18). In line, Thorincroft et al. (2015) reported that anti-stigma group interventions were particularly effective in patients with mental disorders (19). Thornicroft et al. (2009) also demonstrated that establishing social rapport increased the desire to face mental health problems and promote anti-stigma behaviors and participation in anti-stigma campaigns. Their results emphasized the importance of creating conditions to enhance appropriate communications with patients (20).

Some studies show that stigma is more intense in Asian countries compared to developed western countries (21), and this can be due to the fact that in individualist cultures (e.g., Americans, Germans, and Australians), deviation from norms is more easily tolerated compared to collectivist cultures (e.g., Asians, Africans, and Arabs), secondary to cultural diversity and promiscuous liberty (18). In addition, the lack of studies and interventions in nations with low to moderate income could be another reason for different levels of stigma among these countries, including Iran as a developing country with a collectivist culture (18). For example, a study conducted by Pawar et al. (2014) suggested that the Indian society would strongly scold and discriminate against family caregivers and patients with mental disorders and the fact that Indians had no desire to have any kind of relationships with them (22). Barke et al. (2011) reported that, like other South Saharan African countries, Ethiopian people had negative attitudes toward chronic mental diseases (23). It seems that the concept of stigma has different meanings in different societies and that the people of most of the above countries do not possess positive views toward chronic mental disorders such as $\mathrm{AD}$, so patients and their family members are occasionally discriminated against and stigmatized in these nations.

In agreement with the findings of previous studies, our findings indicated that most of our caregivers were under the strain of caregiving, and the educational program was effective in alleviating such a burden. According to Lewis et al. (2009), giving care to a person with AD is a chronic stressful process that undermines caregivers' physical and mental health (24). Therefore, it is essential to improve caregivers' health and empower them for caregiving. Caregivers need to learn the techniques facilitating their coping with caregiving stress. Moreover, educating them about communication skills can reduce their psychological problems and facilitate their coping with caregiving difficulties.

Our findings showed that group discussions reduced AD caregivers' perceived stigma. Psychological interventions can be used to enhance caregivers' abilities and empower them to manage their problems and give effective care to their ill family members, which would alleviate their caregiving stress. Such interventions include, but are not limited to, emotional release, group-based therapies, cognitive therapies, and counseling. On the other hand, educational interventions not only are helpful in enhancing knowledge and problem-solving skills, but also can im- 


\begin{tabular}{|c|c|c|c|}
\hline Variables & Experimental Group & Control Group & Statistical Tests ${ }^{\text {b }}$ \\
\hline Total & $30(100)$ & $31(100)$ & \\
\hline Gender & & & $\chi^{2}=1.501^{c} ; \mathrm{df}=1 ; \mathrm{P}=0.221$ \\
\hline Female & $26(86.7)$ & $23(74.2)$ & \\
\hline Male & $4(13.3)$ & $8(25.8)$ & \\
\hline Number of caregivers & & & $\chi^{2}=3.101^{c} ; \mathrm{df}=2 ; \mathrm{P}=0.212$ \\
\hline One & $18(60)$ & $12(38.7)$ & \\
\hline Two & $5(16.7)$ & $10(32.3)$ & \\
\hline Three and more & $7(23.3)$ & $9(29)$ & \\
\hline Place where the afflicted person lives & & & $\mathrm{P}=0.553^{\mathrm{d}}$ \\
\hline Private home & $24(82.8)$ & $21(70)$ & \\
\hline Caregiver's home & $4(13.8)$ & $7(23.3)$ & \\
\hline Both & $1(3.4)$ & $2(6.7)$ & \\
\hline Length of daily care & & & $P=0.762^{d}$ \\
\hline Less than 24 hours & $3(10.3)$ & $3(13)$ & \\
\hline Entire day-night & $26(89.7)$ & $20(87)$ & \\
\hline Types of the afflicted person's insurance & & & $P=0.162^{d}$ \\
\hline Social security & $8(27.6)$ & $4(18.2)$ & \\
\hline Military & $8(27.6)$ & $6(27.3)$ & \\
\hline Therapeutic services & $9(31)$ & $12(54.5)$ & \\
\hline Others & $4(13.8)$ & 0 & \\
\hline Type of the caregiver's insurance & & & $P=0.378^{d}$ \\
\hline Social security & $9(36)$ & $7(33.3)$ & \\
\hline Military & $7(28)$ & $3(14.3)$ & \\
\hline Therapeutic services & $6(24)$ & $10(47.6)$ & \\
\hline Others & $3(12)$ & $1(4.8)$ & \\
\hline Income & & & $\chi^{2}=1.991^{c} ; \mathrm{df}=2 ; \mathrm{P}=0.158$ \\
\hline Sufficient & $18(60)$ & $13(41.9)$ & \\
\hline Insufficient & $12(40)$ & $18(58.1)$ & \\
\hline
\end{tabular}

${ }^{\mathrm{a}}$ Values are expressed as No. (\%).

${ }^{\mathrm{b}}$ Significance level: $\mathrm{P}<0.05$.

c Pearson's chi-square test.

${ }^{\mathrm{d}}$ Fisher exact test.

prove patients' and their caregivers' quality of lives. Carretero et al. noted that official psychological interventions could alleviate caregivers' perceived stigma and stress, relieve caregiving burden, provide caregivers with comfort, and finally reduce the negative effects of care on afflicted persons (25).

One of the limitations of the present study was that because not considering ethnic diversity in Iran and other countries, these results cannot be generalized to other cities and countries. Considering that stigma is a highly culture-dependent phenomenon, it is recom- mended to conduct similar studies in other regions and among various ethnic subgroups. In these types of studies, cross-contamination between the intervention and control groups can be a possibility, and to avoid this, assessments were first conducted in the control group and then in the trial. Despite random sampling, women existed with a higher proportion among caregivers, which could impair the generalizability of our results. 


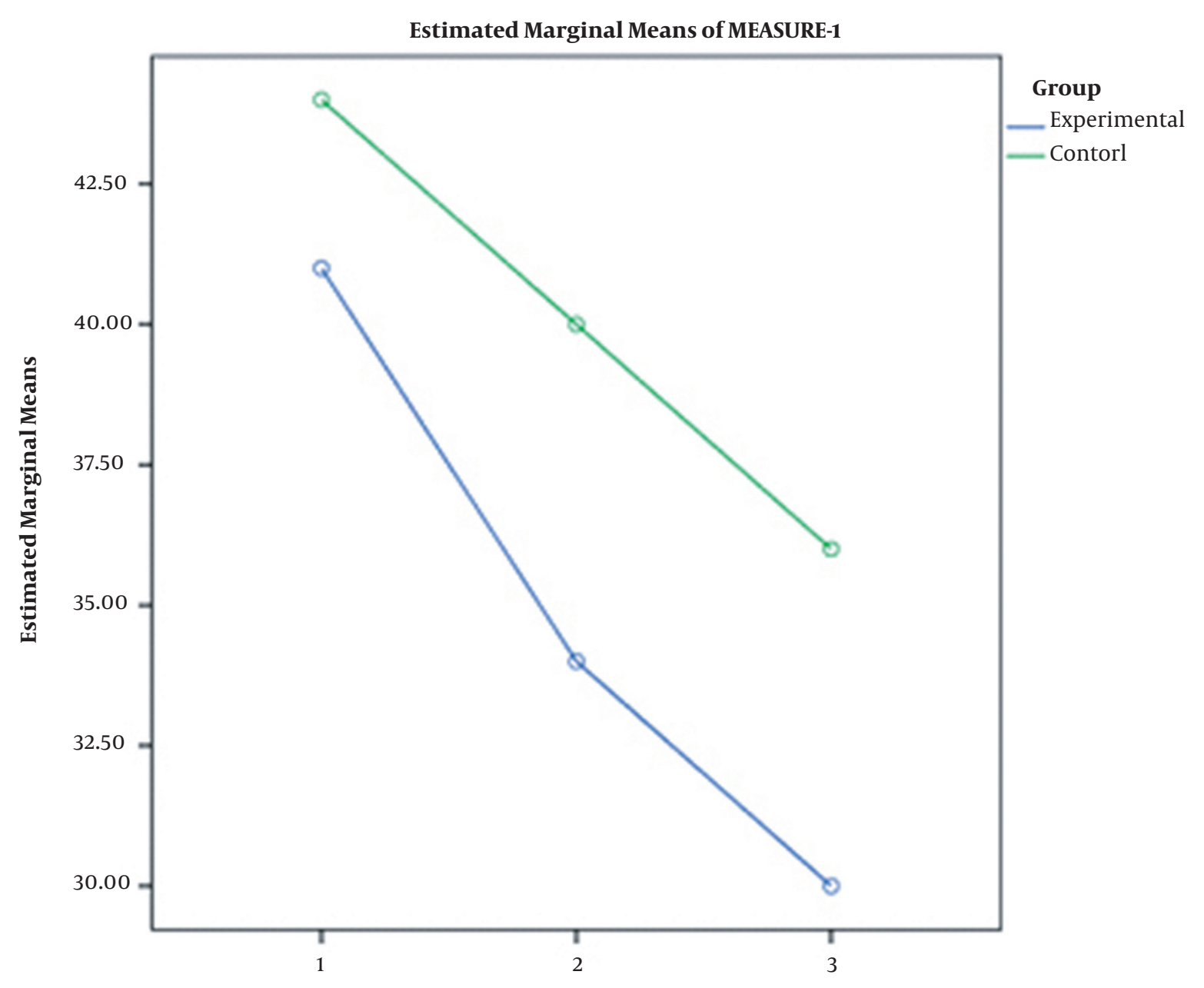

Stigma

Figure 2. Variations in perceived stigma scores in both groups at different measurement time points

\subsection{Conclusion}

The findings of the present study showed the noticeable effects of a group discussion-based educational program on AD family caregivers' perceived stigma. These findings can denote the importance of experience sharing in the psychological well-being of AD caregivers. Group discussion and experience sharing help caregivers learn necessary skills for coping with their own problems.

Health authorities, policymakers, and healthcare providers are recommended to use group discussion and other educational interventions to reduce perceived stigma among AD caregivers. Given the multidimensionality of the concept of perceived stigma, we recommend investigating the effects of other interventions on perceived stigma among the caregivers of patients with $\mathrm{AD}$ and other chronic health conditions in future studies.

\section{Acknowledgments}

The authors wish to express their gratitude to the Research Administration of the Faculty of Nursing and Midwifery of Tehran University of Medical Sciences, Iranian Alzheimer's Association, and all the caregivers who participated in the study. 
Alipour Chermahini M et al.

\begin{tabular}{|c|c|c|c|}
\hline Time & Experimental Group & Control Group & Independent-Sample $t$ Test $^{\mathrm{b}}$ \\
\hline Baseline (T1) & $41.02 \pm 9$ & $44.35 \pm 8.6$ & $\mathrm{t}=1.47 ; \mathrm{df}=59 ; \mathrm{P}=0.146$ \\
\hline $\begin{array}{l}\text { Immediately after intervention } \\
\text { (T2) }\end{array}$ & $34.78 \pm 6.5$ & $40.33 \pm 6.9$ & $\mathrm{t}=3.23 ; \mathrm{df}=59 ; \mathrm{P}<0.001$ \\
\hline One month after intervention (T3) & $30.76 \pm 7.84$ & $36.3 \pm 8.96$ & $\mathrm{t}=2.57 ; \mathrm{df}=59 ; \mathrm{P}=0.013$ \\
\hline \multicolumn{4}{|c|}{ The results of RM ANOVA ${ }^{c}$} \\
\hline Greenhouse test & & $P=0.807$ & \\
\hline Within group & & $\mathrm{F}=34.915 ; \mathrm{P}<0.001 ; \eta^{2}=0.372$ & \\
\hline Between group & & $\mathrm{F}=8.769 ; \mathrm{P}=0.004 ; \eta^{2}=0.129$ & \\
\hline Interaction & & $\mathrm{F}=0.684 ; \mathrm{P}=0.506 ; \eta^{2}=0.011$ & \\
\hline T1 - T2 & & $5.133(2.803,7.463) ; \mathrm{P}<0.001$ & \\
\hline T1 - T3 & & $9.152(5.851,12.453) ; \mathrm{P}<0.001$ & \\
\hline T2 - T3 & & $4.019(1.645,6.392) ; \mathrm{P}<0.001$ & \\
\hline
\end{tabular}

\section{Footnotes}

Authors' Contribution: Conceptualization, Elham Navab, Mahboobeh Alipour, Soroor Parvizy, and Fatemeh Hajibabaee; Writing the original draft, Mahboobeh Alipour, Soroor Parvizy, and Fatemeh Hajibabaee; Data collection, Mahboobeh Alipour; Data analysis, Hamid Haghani; Reviewing the final draft, All authors.

\section{Clinical Trial Registration Code: IRCT201502047212N6}

Conflict of Interests: The authors declare that there is no conflict of interest.

Ethical Approval: This study was approved by the Institutional Review Board of the Tehran University of Medical Sciences, Tehran, Iran (IRB approval number: 94S250802, ethical code: 27804$)$.

Funding/Support: The Deputy of Research and Technology of Tehran University of Medical Sciences financially supported this study.

Informed Consent: Verbal and written informed consent were obtained.

\section{References}

1. Brookmeyer R, Johnson E, Ziegler-Graham K, Arrighi HM. Forecasting the global burden of Alzheimer's disease. Alzheimers Dement. 2007;3(3):186-91. doi: 10.1016/j.jalz.2007.04.381. [PubMed: 19595937].

2. World Health Organization. Dementia cases set to triple by 2050 but still largely ignored. Geneva, Switzerland: World Health Organization; 2012.
3. Mohammadi F, Babaee M. [Effects of participation in support groups on Alzheimer's family caregivers' strain and spiritual wellbeing]. Iranian J Ageing. 2011;6(1). Persian.

4. Zohari S, Khatouni S, Abed Saeidi ZH, Alavi Majd H, Yaghmaei F. [Problems of main caregivers of Alzheimer patients referring to Alzheimer Association in Tehran]. Advances in Nursing and Midwifery (Faculty of Nursing of Midwifery Quarterly). 2006;16(53):64-72. Persian.

5. Abojabel H, Werner P. Exploring family stigma among caregivers of persons with Alzheimer's disease: The experiences of Israeli-Arab caregivers. Dementia (London). 2019;18(1):391-408. doi: 10.1177/1471301216673920. [PubMed: 27885069].

6. Su JA, Chang CC. Association between family caregiver burden and affiliate stigma in the families of people with dementia. Int J Environ Res Public Health. 2020;17(8). doi: 10.3390/ijerph17082772. [PubMed: 32316454]. [PubMed Central: PMC7215659].

7. Goffman E. Stigma: Notes on the management of spoiled identity. London, England: Harmondsworth (Penguin); 2009.

8. Voorend CG, Van Brakel WH, Cross H, Augustine V, Ebenso B. Report of the stigma research workshop for the development of scientific consensus papers and field guidelines on health-related stigma, held in Amsterdam, the Netherlands from 11-14 october 2010. Lepr Rev. 2011;82(2):188-201. [PubMed: 21888143].

9. Brodaty H, Donkin M. Family caregivers of people with dementia. Dialogues Clin Neurosci. 2009;11(2):217-28. [PubMed: 19585957]. [PubMed Central: PMC3181916].

10. Werner P, Heinik J. Stigma by association and Alzheimer's disease. Aging Ment Health. 2008;12(1):92-9. doi: 10.1080/13607860701616325. [PubMed: 18297483].

11. Park S, Park KS. Family stigma: A concept analysis. Asian Nursing Research. 2014;8(3):165-71.

12. Pahlavanzadeh S, Heidari FG, Maghsudi J, Ghazavi Z, Samandari S. The effects of family education program on the caregiver burden of families of elderly with dementia disorders. Iran J Nurs Midwifery Res. 2010;15(3):102-8. [PubMed: 21589771]. [PubMed Central: PMC3093163].

13. Hosseiny RS, Bastani F, Sayahi S, Momen-Abadi H, AlijanpoorAghamaleki M. The effect of educational-counseling program on general health of women as caregivers of patient, with Alzheimerdisease. J Shahrekord Univ Med Sci. 2011;13(5):83-92. 
14. Liu MF. Perceived stigma in caregivers of persons with dementia and its impact on depressive symptoms [dissertation]. Iowa, USA: The University of Iowa; 2011.

15. Erkut S. Developing Multiple Language Versions of Instruments for Intercultural Research. Child Dev Perspect. 2010;4(1):19-24. doi: 10.1111/j.1750-8606.2009.00111.x. [PubMed: 21423824]. [PubMed Central: PMC3060794].

16. Vaghee S, Salarhaji A, Asgharipour N, Chamanzari H. [Effects of psychoeducation on stigma in family caregivers of patients with schizophrenia: A clinical trial]. Evidence Based Care. 2015;5(3):63-76. Persian.

17. Uchino T, Maeda M, Uchimura N. Psychoeducation may reduce self-stigma of people with schizophrenia and schizoaffective disorder. Kurume Med J. 2012;59(1-2):25-31. doi: 10.2739/kurumemedj.59.25. [PubMed: 23257635].

18. Griffiths KM, Carron-Arthur B, Parsons A, Reid R. Effectiveness of programs for reducing the stigma associated with mental disorders. A meta-analysis of randomized controlled trials. World Psychiatry. 2014;13(2):161-75. doi: 10.1002/wps.20129. [PubMed: 24890069]. [PubMed Central: PMC4102289].

19. Thornicroft G, Mehta N, Clement S, Evans-Lacko S, Doherty M, Rose $\mathrm{D}$, et al. Evidence for effective interventions to reduce mental-healthrelated stigma and discrimination. Lancet. 2016;387(10023):1123-32. doi: 10.1016/S0140-6736(15)00298-6. [PubMed: 26410341].
20. Thornicroft G, Brohan E, Rose D, Sartorius N, Leese M; Indigo Study Group. Global pattern of experienced and anticipated discrimination against people with schizophrenia: A cross-sectional survey. Lancet. 2009;373(9661):408-15. doi: 10.1016/S0140-6736(08)61817-6. [PubMed: 19162314].

21. Lauber C, Rossler W. Stigma towards people with mental illness in developing countries in Asia. Int Rev Psychiatry. 2007;19(2):157-78. doi: 10.1080/09540260701278903. [PubMed: 17464793].

22. Pawar AA, Peters A, Rathod J. Stigma of mental illness: A study in the Indian Armed Forces. Med J Armed Forces India. 2014;70(4):354-9. doi: 10.1016/j.mjafi.2013.07.008. [PubMed: 25382910]. [PubMed Central: PMC4223200].

23. Barke A, Nyarko S, Klecha D. The stigma of mental illness in Southern Ghana: Attitudes of the urban population and patients' views. Soc Psychiatry Psychiatr Epidemiol. 2011;46(11):1191-202. doi: 10.1007/s00127010-0290-3. [PubMed: 20872212]. [PubMed Central: PMC3192946].

24. Lewis SL, Miner-Williams D, Novian A, Escamilla MI, Blackwell $\mathrm{PH}$, Kretzschmar JH, et al. A stress-busting program for family caregivers. Rehabil Nurs. 2009;34(4):151-9. doi: 10.1002/j.20487940.2009.tb00271.x. [PubMed: 19583056].

25. Carretero S, Garces J, Rodenas F, Sanjose V. The informal caregiver's burden of dependent people: Theory and empirical review. Arch Gerontol Geriatr. 2009;49(1):74-9. doi: 10.1016/j.archger.2008.05.004. [PubMed: 18597866]. 